\title{
2006-1455: DESIGN, BUILD AND TEST IN SUPPORT OF COMPUTER AIDED
}

DESIGN

\section{John Burkhardt, U.S. Naval Academy}

John Burkhardt received a Bachelors Degree in Civil Engineering from The Cooper Union in New York City. At the University of Illinois Prof. Burkhardt received his Masters of Science and Doctor of Philosophy degree in Theoretical and Applied Mechanics. Prof. Burkhardt is currently on the faculty at the United States Naval Academy in the Mechanical Engineering Department. 


\section{Design, Build and Test in Support of Computer Aided Design}

\section{Introduction}

A senior level project requiring the design, fabrication and testing of an idealized lift hook is discussed. The primary objective of the project is to address the issue of solution accuracy $^{1}$ and its importance in computer aided design (CAD). Secondary objectives of the project are to reinforce the CAD process, introduce the computer numerically controlled (CNC) machining process, and to provide some of the tools and skill helpful to students during their senior capstone design projects.

The primary CAD tools used in the completion of the project are solid modeling and the finite element method (FEM). Critical to the successful application of the FEM, under all circumstances, is the generation of an accurate solution. As defined here accuracy requires the construction of a proper finite element model (e.g. finite element, restraints, loads, etc...), as well as a numerically robust solution. Accuracy must answer two questions:

- Is the computational model sufficiently accurate ${ }^{2}$ ?

- Is the numerical solution sufficiently accurate ${ }^{3,4}$ ?

While it is difficult to determine firm answers to these questions it is important that they are asked. The lift hook project being discussed and its design, build, and test format is constructed to raise awareness of these issues.

\section{Course description}

The lift hook design project is part of the first course in a two course capstone design sequence in mechanical engineering at the United States Naval Academy. The first semester course is structured to have two hours of lecture and two hours of laboratory per week. Course credit is split evenly between computer aided design content (CAD) and the capstone design project.

The CAD portion of the course is project driven covering topics in solid modeling, drafting, finite element analysis, assembly modeling and rapid prototyping. Approximately a third of this time is spent on design projects using finite element analysis. The specific analyses required include rods and beams, frames, linear elastic solids and heat transfer. The last three projects are team assignments. The finite element lecture content has changed from year to year ranging from a miniature finite element course to a just-in-time delivery model. The lecture topics and course materials of relevance to this project included the basic element stiffness approach for rods and trusses, plane stress rectangular finite elements and formal and heuristic approaches to determine solution accuracy and convergence.

\section{Project description}

While all of the finite element projects in the course contain an element of design, build and test the most well developed project, and the one discussed here, concerns the linear elastic behavior of a lift hook. The project requires the design of a lift hook with the greatest stiffness 
per unit weight using the solid modeling and finite element analysis capabilities in IDEAS. After the hook design is finalized the $\mathrm{CNC}$ code required to manufacture the hook is generated, uploaded onto a CNC mill and the hook is manufactured. The resulting hook is then tested under uniaxial loading. The accuracy of the team's finite element analysis is then determined by comparing the experimental and finite element analysis predictions.

The project is assigned to teams of two students who are to design, build and test a simplified lift hook. The lift hook is to be constructed from $1 / 2$-in PVC sheeting. Each lift hook is required to fit within a 6"x 10 " rectangle. Two circular cuts through the thickness of the hook are required. A 1" diameter hole is required at the "top" of the hook to mimic the lift hook's attachment to a crane and to provide for fixturing during testing. A second fixturing hole, 2 " in diameter and 5" below the center of the 1" hole, is required and will form the bowl shaped portion of the hook. A schematic of this basic lift hook "blank" is shown in Figure 1. An additional cut, under the control of the designer, is required to produce a slot through the side of the hook to connect with the 2 " hole. In combination the slot and the hole produce the open throat "hook" portion of the lift hook. At a minimum the lower slot must allow a 2 " pin to pass through and seat in the 2 " diameter hole. Any other material can be removed from the hook blank provided the hook remains a uniform thickness. Hooks with variable thickness are not allowed because of the complications it would add to the machining process. A generic lift hook is shown in Figure 2.

It is required that solid modeling and finite element analysis be used to guide and improve the design process. Initially, each team member is required to develop an independent lift hook design using a combination of solid modeling and finite element techniques. Each student is encouraged to consider several design iterations and through observation most do. The individual design within each team with the greatest stiffness per unit weight will become the team's hook. At this point further iterations are encouraged

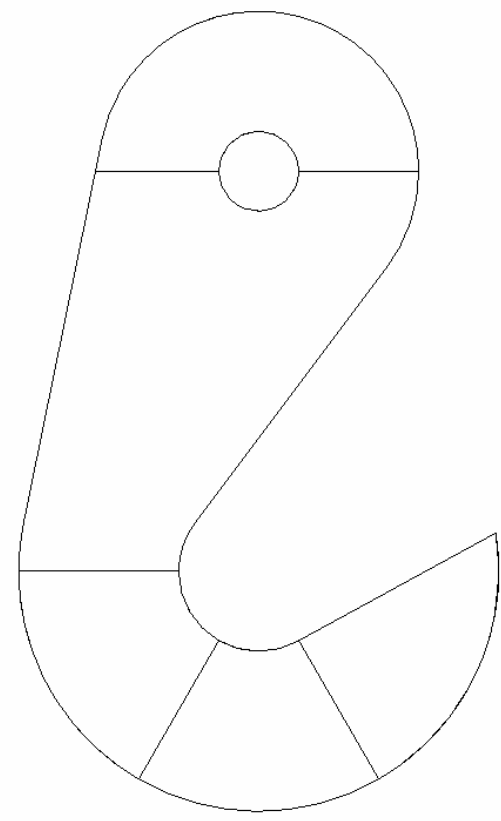

Figure 2 Generic lift hook design 
using the insights gain during the initial design phase.

Idealized restraints and loading conditions are provided to the students. For simplicity the upper half of the top through hole is assigned fixed displacements and rotations. Loading is modeled as a $1000 \mathrm{lb}$ uniformly distributed pressure load, Figure 3. Specifying these details does not eliminate the student's role in producing an accurate finite element. Rather, it requires the student to interpret the effects of the provided boundary conditions on the resulting solution. Often these effects are spurious, unrelated to the true physics of the problem. For example, applied loads and boundary conditions often create singular-like stresses. These stresses arise regularly in the lift hook design and provide an opportunity to discuss the shortcomings and advantages of each of the provided boundary conditions. It is also specified that all of the finite element analyses of initial designs should use a mesh size, $\mathrm{h}$, equal to 0.10 inches. The effect mesh size on numerical accuracy is addressed during the design of each team's final hook.

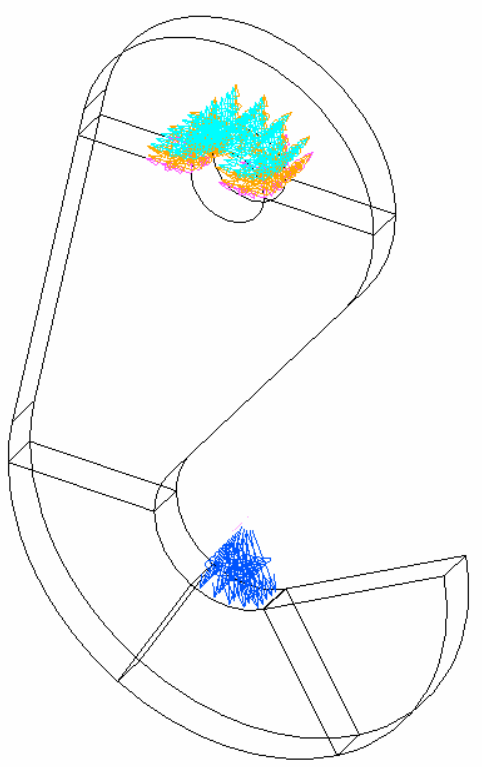

Figure 1 Lift hook boundary conditions

It is required that the team hook with the greatest stiffness per unit weight be modified and improved using a refined finite element analysis. Specifically, each team is required to produce a finite element model using variable mesh densities. A previous tutorial provided an introduction to formal convergence studies and the effect of element size on solution accuracy. In this project convergence is handled heuristically by requiring that the mesh densities in the area of the largest stress gradient be refined until the unaveraged stress contours change smoothly across the hook. An example of smooth stress contours is shown in Figure . This figure shows the predicted unaveraged von Mises stresses for the hook shown in Figure 2 and Figure 3. When "converged" a final estimate of the hook's stiffness per unit weight and an estimated failure location are documented.

The manufacture of each hook is accomplished using an exported STEP file of the solid model. The STEP file is used to drive a CNC end mill. The final hook from each team is then tested on a screw driven load frame. Load deflection data is recorded and the failure location is photographically documented. From the load deflection data estimates of the hook's experimental stiffness are made and compared to those predicted by the finite element

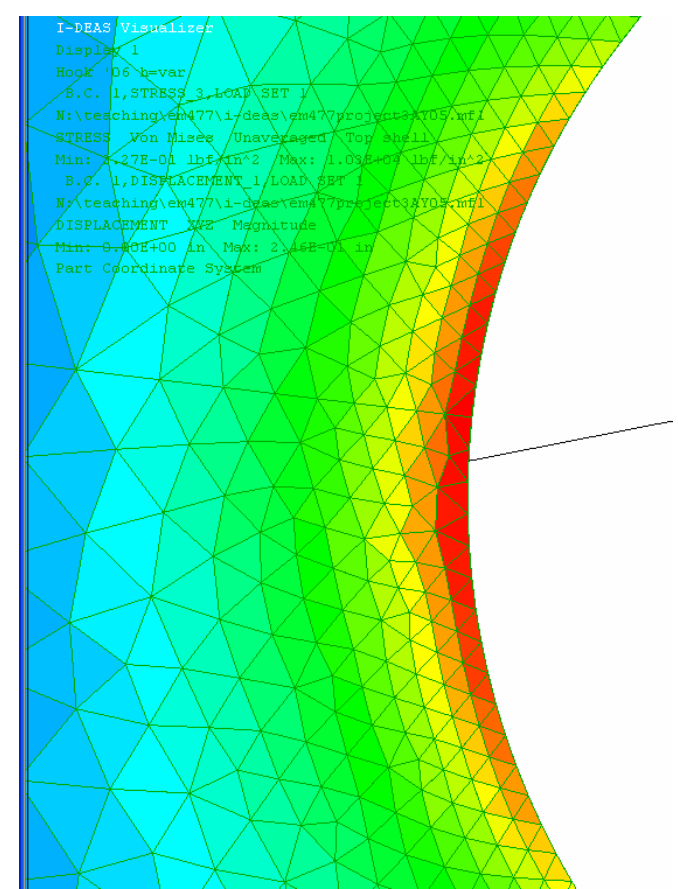

Figure 4 "Smooth" unaveraged stress contours 
analysis. This comparison is used as the accuracy measure for each team's finite element analysis.

\section{Project results}

A team hook from the fall semester of 2005 is shown below because of its relatively interesting shape and its failure. In this instance the predicted failure location (black marker X) and the observed failure location are almost identical.

\section{Conclusion}

The lift hook project has had a positive impact on several aspects of the CAD course. Pedagogically the project has been very successful at introducing and demonstrating the importance of accuracy in finite element analysis. It has also, however, been very effective at raising student interest. Across two semesters it is clear that the students love the competitive aspects of the project and, most notably, love the opportunity to get out from behind their monitors and touch, feel and test something that they have designed. This is supported by end-ofsemester comments in which students consistently indicate that the $\mathrm{CNC}$ and testing portions of the project are fun and help them learn the material.

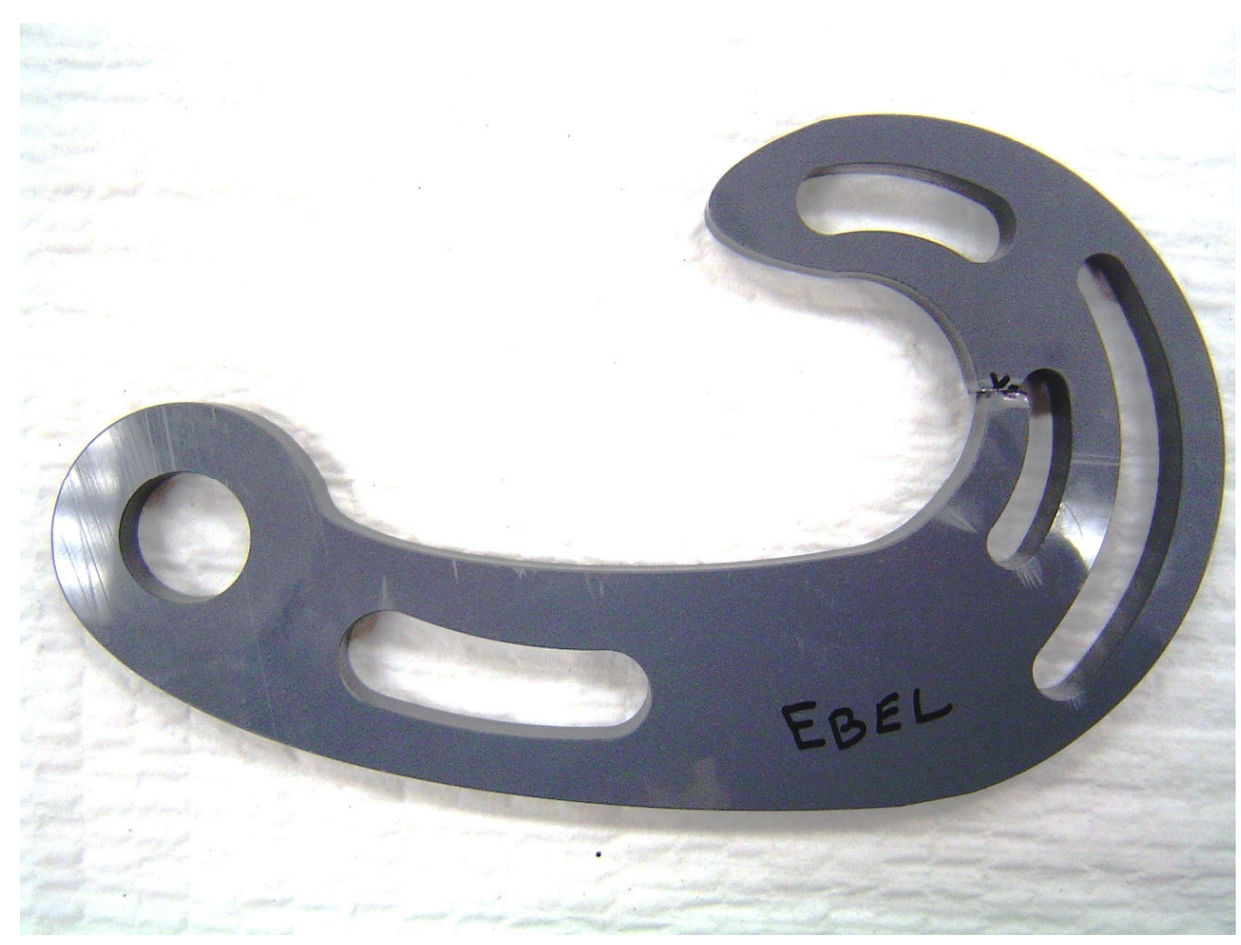

Figure 2 Test hook example 


\section{Endnotes}

${ }^{1}$ Adams, V. and A. Askenazi, Building Better Products with Finite Element Analysis, Onword Press: Santa Fe, 1999, p. 99.

${ }^{2}$ Verification: solving the right equations, e.g. plane stress versus plane strain.

${ }^{3}$ Validation: solving the equations right, e.g. how accurate is the solution in the neighborhood of a discontinuity.

${ }^{4}$ P.J. Roache, Verification and Validation in Computational Science and Engineering (Albuquerque: Hermosa Publishers, 1998). 